\title{
Permanence and partial extinction in a delayed three-species food chain model with stage structure and time-varying coefficients
}

\author{
Huanyan Xi, Lihong Huang, Yuncheng Qiao, Huaiyu Li, Chuangxia Huang* \\ Department of Mathematics and Statistics, Changsha University of Science and Technology, 410114, Changsha, P. R. China.
}

Communicated by M. Bohner

\begin{abstract}
By taking full consideration of maturity $\left(\tau_{1}\right.$ represents the maturity of predator and $\tau_{2}$ represents the maturity of top predator) and the effects of environmental parameters, a new delayed three-species food chain model with stage structure and time-varying coefficients is established. With the help of the comparison theorem and the technique of mathematical analysis, the positivity and boundedness of solutions of the model are investigated. Furthermore, some sufficient conditions on the permanence and partial extinction of the system are derived. Some interesting findings show that the delays have great impacts on the permanence for the system. More precisely, if $\tau_{2} \in(n,+\infty)$, then the system is partially extinct: on one hand, if $\tau_{1} \in\left(0, n_{1}\right)$ and $\tau_{2} \in(n,+\infty)$, then the prey and predator species will coexist, i.e., both the prey and predator species are always permanent, yet the top predator species will go extinct eventually. On the other hand, if $\tau_{1} \in\left(n_{4},+\infty\right)$ and $\tau_{2} \in(n,+\infty)$, where $n_{4}$ is greater than $n_{1}$, then all predator species will become extinct eventually. Numerical simulations are great well agreement with the theoretical results. (c) 2017 All rights reserved.
\end{abstract}

Keywords: Food chain model, delay, stage structure, permanence, extinction.

2010 MSC: 03C45, 34D05.

\section{Introduction}

The dynamic interactions between the predator and their prey have long been one of the dominant themes in ecology [3]. The classical predator-prey type model

$$
\left\{\begin{array}{l}
\dot{x}(t)=x(t)\left[r_{1}-a_{11} x(t)-a_{12} y(t)\right] \\
\dot{y}(t)=y(t)\left[-r_{2}+a_{21} x(t)-a_{22} y(t)\right]
\end{array}\right.
$$

has been extensively investigated $[11,13,28,30]$, where $x(t)$ and $y(t)$ can be interpreted as the population densities of prey and predator at time $t$, respectively; $r_{1}>0$ denotes intrinsic growth rate of prey, $r_{2}>0$ denotes the death rate of predator; the parameters $a_{i j}(i, j=1,2)$ are all positive constants.

\footnotetext{
*Corresponding author

Email addresses: huanyanxii@126.com (Huanyan Xi), lhhuang@csust.edu.cn (Lihong Huang), lyuqyc@163.com (Yuncheng Qiao), lee1012116@126.com (Huaiyu Li), cxiahuang@126.com (Chuangxia Huang)

doi:10.22436/jnsa.010.12.05
} 
We note that the stage structure and the maturation of the species are ignored and each individual prey has the same chance of suffering attacks from the predators in system (1.1). However, there are many species, such as some mammalian populations and some amphibious animals, whose individuals have a life history that take them through two stages, immature and mature in the natural world [7]. On the other hand, time delay due to the gestation and the maturation of species is a common phenomenon, which reflects a delay birth of immatures and a reduced survival of immatures to their maturity. And the time delays play an important role in the dynamic behaviors of system, such as population system, economic system, epidemic model, neural network system, etc., (for more details about these studies, we refer to $[16-19,21,24])$. Thus, it is of great importance to incorporate the stage-structured and time delays into the predator-prey models.

Up to 1990, because of the seminal work by Aiello and Freedman [1], species growth models with stage structure have received a great amount of attention. It assumes that an average age to maturity which appears as a constant time delay reflecting a delayed birth of immatures and a reduced survival of immatures to their maturity. The single species growth model with stage structure takes the form as below

$$
\left\{\begin{array}{l}
\dot{x}_{i}(t)=\alpha x_{m}(t)-\gamma x_{i}(t)-\alpha e^{-\gamma \tau} x_{m}(t-\tau), \\
\dot{x}_{m}(t)=\alpha e^{-\gamma \tau} x_{m}(t-\tau)-\beta x_{m}^{2}(t), \quad t>\tau,
\end{array}\right.
$$

where $x_{i}(t)$ denotes the immature population density, $x_{m}(t)$ represents the mature population density, $\alpha>0$ represents the birth rate, $\gamma>0$ is the immature death rate, $\beta>0$ is the mature death and overcrowding rate, $\tau \geqslant 0$ is the time to maturity. The term $e^{-\gamma \tau} x_{m}(t-\tau)$ represents the immatures who were born at time $t-\tau$ and survive at time $t$. After then, different types stage-structured models have been investigated extensively by many researchers and some significant works were implemented. For instance, $\mathrm{Xu}$ [32] incorporated stage structure for predator into the system and investigated the effect of the duration time of immature predator on the dynamics of the system. Considering the stage structure for both prey and predator, Chen et al. [6] established an autonomous predator-prey system with stagestructure for all the species and investigated the global stability of the system. The model can be described as the following

$$
\left\{\begin{array}{l}
\dot{x_{1}}(t)=r_{1} x_{2}(t)-d_{11} x_{1}(t)-r_{1} e^{-d_{11} \tau_{1}} x_{2}\left(t-\tau_{1}\right), \\
\dot{x_{2}}(t)=r_{1} e^{-d_{11} \tau_{1}} x_{2}\left(t-\tau_{1}\right)-d_{12} x_{2}(t)-b_{1} x_{2}^{2}(t)-c_{1} x_{2}(t) y_{2}(t), \\
\dot{y} 1(t)=r_{2} y_{2}(t)-d_{22} y_{1}(t)-r_{2} e^{-d_{22} \tau_{2}} y_{2}\left(t-\tau_{2}\right), \\
\dot{y}_{2}(t)=r_{2} e^{-d_{22} \tau_{2}} y_{2}\left(t-\tau_{2}\right)-d_{21} y_{2}(t)-b_{2} y_{2}^{2}(t)+c_{2} x_{2}(t) y_{2}(t),
\end{array}\right.
$$

where $x_{1}(t)$ and $x_{2}(t)$ represent the densities of the immature and mature prey species at time $t$, respectively; $y_{1}(t)$ and $y_{2}(t)$ denote the immature and mature population densities of predator species at time $t$, respectively. Also, the parameters $r_{1}, r_{2}, c_{1}, c_{2}, d_{11}, d_{12}, d_{21}, d_{22}$ and $b_{i}(i=1,2)$ are positive constants, $\tau_{1}$ and $\tau_{2}$ are nonnegative constants. More recent interesting results with stage-structure models can be found in $[10,14,15,26,33]$.

In an ecological system, several species tend to be linked together and constitute a food-chain system [29]. As pointed out by Charles [5], a single food chain should have at least three links to be complete plant-herbivore-carnivore. An example of a three-link chain occurring on the North American Great Plains is pronghorns eat grasses for food, and coyotes eat pronghorns. It is important and meaningful to study dynamical behavior of the food-chain system. Based on this, the Lotka-Volterra type three-species food chain model was proposed and investigated by Baek and Lee [2]. The three-species food chain model takes the form as below

$$
\left\{\begin{array}{l}
\dot{x}(t)=x(t)(a-b x(t)-c y(t)) \\
\dot{y}(t)=y(t)\left(-d_{1}+c_{1} x(t)-e_{1} z(t)\right), \\
\dot{z}(t)=z(t)\left(-d_{2}+e_{2} y(t)\right)
\end{array}\right.
$$

where $x(t), y(t), z(t)$ denote the population densities of the lowest-level prey, mid-level predator and top 
predator at time $t$, respectively; the constant $a>0$ is called the intrinsic growth rate of the prey species; $\mathrm{b}>0$ measures the intraspecific competition of the prey; $c>0$ and $e_{1}>0$ are the predation rate per capita of the mid-level and top predator, respectively; $c_{1}>0$ and $e_{2}>0$ represent the conversion rates of the lowest-level prey to the mid-level predator and the mid-level predator to the top one, respectively; $d_{1}>0$ and $d_{2}>0$ denote the death rate of the mid-level and top predator, respectively.

To date, a great deal of results on three-species food chain models have been reported in the literature. In [20], the stability and bifurcation analysis of a stage structured predator-prey model with time delay were investigated. Yongzhen et al. [34] formulated a three-species ecosystem with a delay digestion process and Holling functional response, the dynamical behaviors of the system are studied. The stability loss arisen from delay in a three-dimensional competition model were discussed by Boudjellaba and Sari [4]. In [25], Patra et al. considered the boundedness and stability criteria in a delayed food chain model with Michaelis-Menten type ratio-dependent functional responses. A three species prey-predator competition model with effect of stochastic perturbation was studied by Das et al. [9]. To a large extent, the existing literature on theoretical studies of three-species food chain models is predominantly concerned without time-varying coefficients and stage structure. Literature dealing with both the time-varying coefficients and the stage structure for the species appears to be scarce. As pointed out by Cushing [8], these biological and environmental parameters are always time-varying, which is due to the effects of seasonal weather, food supply, mating habits, hunting or harvesting seasons, etc.

The concepts of permanence and extinction, which respectively meaning that the population system will survive or die out in the future. Just because of the biological meaning, the permanence and extinction analysis for dynamic systems have attracted considerable attention, and many interesting results were presented (for example, see [12, 22, 27, 31, 35]). Different from the two dimensional predator-prey model, partial extinction instead of extinction is the key characteristic property for the food chain model. For a three-species food chain model, partial extinction means that both the prey and predator species are permanent, the top predator species will go extinct eventually, or all predator species become extinct.

Enlightened by the above discussions, according to biological significance, food chain model commonly considers plant as the prey, without introducing age levels for the prey (plant) is reasonable in some sense, therefore, in this paper, we intend to investigate the permanence and partial extinction of a new delayed three-species food chain model with time-varying coefficients and stage structure for both predator and top predator.

We build up the following stage-structured food chain model firstly:

$$
\left\{\begin{array}{l}
\dot{x}(t)=x(t)\left[\alpha_{1}(t)\left(1-\frac{x(t)}{k}\right)-c_{1}(t) y_{2}(t)\right] \\
\dot{y}_{1}(t)=\alpha_{2}(t) x(t) y_{2}(t)-d_{11} y_{1}(t)-\alpha_{2}\left(t-\tau_{1}\right) e^{-d_{11} \tau_{1}} x\left(t-\tau_{1}\right) y_{2}\left(t-\tau_{1}\right) \\
\dot{y}_{2}(t)=\alpha_{2}\left(t-\tau_{1}\right) e^{-d_{11} \tau_{1}} x\left(t-\tau_{1}\right) y_{2}\left(t-\tau_{1}\right)-d_{12} y_{2}(t)-\beta_{1}(t) y_{2}^{2}(t)-c_{2}(t) y_{2}(t) z_{2}(t), \\
\dot{z}_{1}(t)=\alpha_{3}(t) y_{2}(t) z_{2}(t)-d_{21} z_{1}(t)-\alpha_{3}\left(t-\tau_{2}\right) e^{-d_{21} \tau_{2}} y_{2}\left(t-\tau_{2}\right) z_{2}\left(t-\tau_{2}\right), \\
\dot{z}_{2}(t)=\alpha_{3}\left(t-\tau_{2}\right) e^{-d_{21} \tau_{2}} y_{2}\left(t-\tau_{2}\right) z_{2}\left(t-\tau_{2}\right)-d_{22} z_{2}(t)-\beta_{2}(t) z_{2}^{2}(t)
\end{array}\right.
$$

The initial conditions for system (1.2) take the form as below

$$
\begin{gathered}
x(\theta)=\phi(\theta)>0, \quad y_{\mathfrak{i}}(\theta)=\varphi_{\mathfrak{i}}(\theta)>0, \quad z_{\mathfrak{i}}(\theta)=\psi_{\mathfrak{i}}(\theta)>0, \\
\phi(0)>0, \quad \varphi_{\mathfrak{i}}(0)>0, \quad \psi_{\mathfrak{i}}(0)>0, \quad i=1,2, \quad \theta \in[-\tau, 0],
\end{gathered}
$$

where $\tau=\max \left\{\tau_{1}, \tau_{2}\right\},\left(\phi(\cdot), \varphi_{1}(\cdot), \varphi_{2}(\cdot), \psi_{1}(\cdot), \psi_{2}(\cdot)\right) \in C\left([-\tau, 0], R_{+0}^{5}\right)$, the Banach space of continuous functions mapping the interval $[-\tau, 0]$ into $R_{+0}^{5}$, and $R_{+0}^{5}=\left\{\left(x_{1}, x_{2}, x_{3}, x_{4}, x_{5}\right): x_{i} \geqslant 0, i=1,2,3,4,5\right\}$.

For continuity of the initial conditions, we further desire

$$
\begin{aligned}
& y_{1}(0)=\int_{-\tau_{1}}^{0} \alpha_{2}(s) \phi(s) \varphi_{2}(s) e^{d_{11} s} d s \\
& z_{1}(0)=\int_{-\tau_{2}}^{0} \alpha_{3}(s) \varphi_{2}(s) \psi_{2}(s) e^{d_{21} s} d s
\end{aligned}
$$


In the following, we introduce the parameters and a brief sketch of the construction of the model.

\begin{tabular}{l|l}
\hline \hline Parameter & Description \\
\hline$\alpha_{1}(t)$ & Intrinsic growth rate of the prey \\
$k$ & Environmental carrying capacity of the prey \\
$c_{1}(t)$ & Capture rate of the mature predator \\
$\frac{\alpha_{2}(t)}{c_{1}(t)}$ & Conversion rate of nutrients into the reproduction of the mature predator \\
$c_{2}(t)$ & Capture rate of the mature top predator \\
$\frac{\alpha_{3}(t)}{c_{2}(t)}$ & Conversion rate of nutrients into the reproduction of the mature top predator \\
$d_{11}$ & Death rate of the immature predator \\
$d_{12}$ & Death rate of the mature predator \\
$d_{21}$ & Death rate of the immature top predator \\
$d_{22}$ & Death rate of the mature top predator \\
$\beta_{1}(t)$ & Intra-specific competition rate of the mature predator species \\
$\beta_{2}(t)$ & Intra-specific competition rate of the mature top predator species \\
$\tau_{1}$ & Maturity for the predator species \\
$\tau_{2}$ & Maturity for the top predator species \\
\hline \hline
\end{tabular}

Table 1: Parameters table for systems (1.2).

(A1) There are three populations, namely, the prey species whose population density is denoted by $x(t)$, the predator whose immature and mature population densities are $y_{1}(t)$ and $y_{2}(t)$, respectively; the top predator whose immature and mature population densities are described by $z_{1}(t)$ and $z_{2}(t)$, respectively.

(A2) $\alpha_{i}(t), c_{j}(t)$ and $\beta_{j}(t)(i=1,2,3 ; j=1,2)$ are continuous and bounded above and below by positive constants.

(A3) In absence of predation, the prey population grow according to logistic laws of growth with intrinsic growth rates $\alpha_{1}(t)$, and the carrying capacity is $k$.

(A4) The mature predator consumes the prey with $c_{1}(t) x(t) y_{2}(t)$ and contributes to its immature population growth rate $\alpha_{2}(t) x(t) y_{2}(t)$. The mature top predator consumes the mature predator with $c_{2}(t) y_{2}(t) z_{2}(t)$ and contributes to its immature population growth rate $\alpha_{3}(t) y_{2}(t) z_{2}(t)$.

(A5) Mortality rate of predator is assumed to be proportional to the existing population. We also consider the density dependent mortality rate of the consumer species as $\beta_{1}(t) y_{2}^{2}(t)$ and $\beta_{2}(t) z_{2}^{2}(t)$. If there is some other factor (other than food) which becomes limiting at high population densities, the self limitation will occur.

(A6) The term $\alpha_{2}\left(t-\tau_{1}\right) e^{-d_{11} \tau_{1}} x\left(t-\tau_{1}\right) y_{2}\left(t-\tau_{1}\right)$ represents the number of immature predators that were born at time $t-\tau_{1}$ which still survive at time $t$ and are transferred from the immature stage to the mature stage at time $t$. The term $\alpha_{3}\left(t-\tau_{2}\right) e^{-d_{21} \tau_{2}} z_{2}\left(t-\tau_{2}\right) y_{2}\left(t-\tau_{2}\right)$ represents the number of top immature predators that were born at time $t-\tau_{2}$ which still survive at time $t$ and are transferred from the immature stage to the mature stage at time $t$.

The remainder of this article is organized as follows. In Section 2, we present the basic notations and preliminary lemmas. The positivity and boundedness of the solutions to system (1.2) are investigated in Section 3. In Section 4, we derive a set of sufficient conditions for the permanence and partial extinction of system (1.2) by using the comparison theorem. One illustrative example and simulations are shown in Section 5. The paper concludes with a brief summary in Section 6. 


\section{Preliminaries}

Throughout this paper, for convenience of our statement, we adopt the notations:

$$
f^{i}=\inf _{t \in R} f(t), \quad f^{s}=\sup _{t \in R} f(t),
$$

where $f(t)$ is a bounded continuous function defined on $R$.

Definition 2.1. System (1.2) is permanent, if there exist positive constants $m_{x}, m_{y}, m_{z}, M_{x}, M_{y}$ and $M_{z}$, such that each solution $\left(x(t), y_{1}(t), y_{2}(t), z_{1}(t), z_{2}(t)\right)$ of system (1.2) satisfies

$$
\begin{gathered}
0<m_{x} \leqslant \lim _{t \rightarrow+\infty} \inf x(t) \leqslant \lim _{t \rightarrow+\infty} \sup x(t) \leqslant M_{x}, \\
0<m_{y} \leqslant \lim _{t \rightarrow+\infty} \inf y_{i}(t) \leqslant \lim _{t \rightarrow+\infty} \sup y_{i}(t) \leqslant M_{y}, \quad(i=1,2), \\
0<m_{z} \leqslant \lim _{t \rightarrow+\infty} \inf z_{i}(t) \leqslant \lim _{t \rightarrow+\infty} \sup z_{i}(t) \leqslant M_{z}, \quad(i=1,2) .
\end{gathered}
$$

Otherwise, system (1.2) is non-permanent.

Lemma 2.2 ([32]). Consider the following equation

$$
\dot{u}(t)=a u(t-\tau)-b u(t)-c u^{2}(t),
$$

where $\mathrm{a}, \mathrm{b}, \mathrm{c}$ and $\tau$ are positive constants, $\mathrm{u}(\mathrm{t})>0$ for $\mathrm{t} \in[-\tau, 0]$, we have:

(i) If $\mathrm{a}>\mathrm{b}$, then $\lim _{\mathrm{t} \rightarrow+\infty} \mathrm{u}(\mathrm{t})=(\mathrm{a}-\mathrm{b}) \mathrm{c}^{-1}$.

(ii) If $\mathrm{a}<\mathrm{b}$, then $\lim _{\mathrm{t} \rightarrow+\infty} \mathrm{u}(\mathrm{t})=0$.

Lemma 2.3 ([23]). Consider the following equation

$$
\dot{u}(t)=u(t)\left[d_{1}-d_{2} u(t)\right],
$$

where $\mathrm{d}_{2}>0$. We have:

(i) If $\mathrm{d}_{1}>0$, then $\lim _{\mathrm{t} \rightarrow+\infty} \mathrm{u}(\mathrm{t})=\mathrm{d}_{1} \mathrm{~d}_{2}^{-1}$.

(ii) If $\mathrm{d}_{1}<0$, then $\lim _{\mathrm{t} \rightarrow+\infty} \mathrm{u}(\mathrm{t})=0$.

\section{Positivity and boundedness of solutions}

In this section, we will prove that the solutions of system (1.2) are positive and ultimately bounded.

Theorem 3.1. Solutions of system (1.2) with initial conditions (1.3), (1.4) and (1.5) are positive for all $\mathrm{t} \geqslant 0$.

Proof. Let $\left(x(t), y_{1}(t), y_{2}(t), z_{1}(t), z_{2}(t)\right)$ be a solution of system (1.2) with initial conditions (1.3), (1.4) and (1.5). We derive from the first equation of system (1.2) that

$$
x(t)=x(0) e^{\int_{0}^{t}\left[\alpha_{1}(s)\left(1-\frac{x(s)}{k}\right)-c_{1}(s) y_{2}(s)\right] d s}>0, \quad \text { for } t \geqslant 0 .
$$

Next we show $y_{2}(t)>0$ for $t \geqslant 0$. Otherwise, noting that $y_{2}(t)=\varphi_{2}(t)>0$ for $-\tau_{1} \leqslant t \leqslant 0$, then there exist a $t_{1}>0$, such that $y_{2}\left(t_{1}\right)=0$. 
Denoting $t_{0}=\inf \left\{t>0: y_{2}(t)=0\right\}$, then $t_{0}>0$. From system (1.2), we have

$$
\dot{y}_{2}\left(t_{0}\right)=\left\{\begin{array}{cr}
\alpha_{2}\left(t_{0}-\tau_{1}\right) e^{-d_{11} \tau_{1}} \phi\left(t_{0}-\tau_{1}\right) \varphi_{2}\left(t_{0}-\tau_{1}\right), & 0 \leqslant t_{0} \leqslant \tau_{1}, \\
\alpha_{2}\left(t_{0}-\tau_{1}\right) e^{-d_{11} \tau_{1}} \chi\left(t_{0}-\tau_{1}\right) y_{2}\left(t_{0}-\tau_{1}\right), & t_{0}>\tau_{1} .
\end{array}\right.
$$

Hence $\dot{y}_{2}\left(t_{0}\right)>0$. But by the definition of $t_{0}, \dot{y}_{2}\left(t_{0}\right) \leqslant 0$. This is a contradiction. Thus $y_{2}(t)>0$ for $t \geqslant 0$. Similarly, we can prove that $z_{2}(t)>0$ for $t \geqslant 0$.

By (1.4) and the second equation of system (1.2), we obtain

$$
y_{1}(t)=\int_{t-\tau_{1}}^{t} \alpha_{2}(s) e^{-d_{11}(t-s)} \chi(s) y_{2}(s) d s>0, \quad \text { for } t \geqslant 0
$$

With initial condition (1.5), we derive from the fourth equation of system (1.2) that

$$
z_{1}(t)=\int_{t-\tau_{2}}^{t} \alpha_{3}(s) e^{-d_{21}(t-s)} y_{2}(s) z_{2}(s) d s>0, \quad \text { for } t \geqslant 0 .
$$

Hence $x(t)>0, y_{i}(t)>0, z_{i}(t)>0(i=1,2)$ for all $t \geqslant 0$. This completes the proof.

Theorem 3.2. Positive solutions of system (1.2) with initial conditions (1.3), (1.4) and (1.5) are ultimately bounded.

Proof. Suppose $\left(x(t), y_{1}(t), y_{2}(t), z_{1}(t), z_{2}(t)\right)$ is any positive solution of system (1.2) with initial conditions (1.3), (1.4) and (1.5). We define

$$
V(t)=\alpha_{2}^{s} x(t)+c_{1}^{i} y_{1}(t)+c_{1}^{i} y_{2}(t)+\frac{c_{1}^{i} c_{2}^{i}}{\alpha_{3}^{s}} z_{1}(t)+\frac{c_{1}^{i} c_{2}^{i}}{\alpha_{3}^{s}} z_{2}(t)
$$

Then

$$
\begin{aligned}
\dot{V}(t)+b V(t) \leqslant & \alpha_{2}^{s}\left(\alpha_{1}^{s}+b\right) x(t)-\frac{\alpha_{1}^{s} \alpha_{2}^{s}}{k} x^{2}(t)+c_{1}^{i}\left(b-d_{11}\right) y_{1}(t)+c_{1}^{i}\left(b-d_{12}\right) y_{2}(t) \\
& +\frac{c_{1}^{i} c_{2}^{i}}{\alpha_{3}^{s}}\left(b-d_{21}\right) z_{1}(t)+\frac{c_{1}^{i} c_{2}^{i}}{\alpha_{3}^{s}}\left(b-d_{22}\right) z_{2}(t)-c_{1}^{i} \beta_{1}^{i} y_{2}^{2}(t) \\
\leqslant & \alpha_{2}^{s}\left(\alpha_{1}^{s}+b\right) x(t)-\frac{\alpha_{1}^{s} \alpha_{2}^{s}}{k} x^{2}(t) \\
\leqslant & \frac{k \alpha_{2}^{s}\left(\alpha_{1}^{s}+b\right)^{2}}{4 \alpha_{1}^{s}}
\end{aligned}
$$

where $b=\min \left\{d_{11}, d_{12}, d_{21}, d_{22}\right\}$. Thus, we have

$$
\mathrm{V}(\mathrm{t}) \leqslant\left[\mathrm{V}(0)-\frac{k \alpha_{2}^{\mathrm{s}}\left(\alpha_{1}^{\mathrm{s}}+\mathrm{b}\right)^{2}}{4 \alpha_{1}^{\mathrm{s}} \mathrm{b}}\right] e^{-\mathrm{bt}}+\frac{k \alpha_{2}^{\mathrm{s}}\left(\alpha_{1}^{\mathrm{s}}+\mathrm{b}\right)^{2}}{4 \alpha_{1}^{\mathrm{s}} \mathrm{b}} .
$$

Choose a positive constant $M$ satisfying $M>\frac{k \alpha_{2}^{s}\left(\alpha_{1}^{s}+b\right)^{2}}{4 \alpha_{1}^{s} b}$. Then there exists a $t_{0}>0$ such that $x(t)<M$, $y_{i}(t)<M$ and $z_{j}(t)<M(i, j=1,2)$, for $t>t_{0}$. This completes the proof.

\section{Permanence and partial extinction}

In this section, we will discuss the permanence and partial extinction of system (1.2).

Theorem 4.1. Let $\mathrm{n}_{1}=\frac{1}{\mathrm{~d}_{11}} \ln \frac{\alpha_{2}^{i} x^{\mathrm{i}}}{\mathrm{d}_{12}+\mathrm{c}_{2}^{\mathrm{s}} z_{2}^{\mathrm{s}}}, \mathrm{n}_{2}=\frac{1}{\mathrm{~d}_{21}} \ln \frac{\alpha_{3}^{\mathrm{i}} y_{2}^{i}}{\mathrm{~d}_{22}}$. If $\tau_{1} \in\left(0, \mathrm{n}_{1}\right), \tau_{2} \in\left(0, \mathrm{n}_{2}\right)$ and $\alpha_{1}^{\mathrm{i}}-\mathrm{c}_{1}^{\mathrm{s}} \mathrm{y}_{2}^{\mathrm{s}}>0$, then system (1.2) is permanent. 
Proof. Let $\left(x(t), y_{1}(t), y_{2}(t), z_{1}(t), z_{2}(t)\right)$ be any positive solution of system (1.2) for $t \geqslant 0$. By $\tau_{1} \in\left(0, n_{1}\right)$ and $\tau_{2} \in\left(0, n_{2}\right)$, one can obtain that $\alpha_{2}^{i} e^{-d_{11} \tau_{1}} \chi^{i}-d_{12}-c_{2}^{s} z_{2}^{s}>0$ and $\alpha_{3}^{i} e^{-d_{21} \tau_{2}} y_{2}^{i}-d_{22}>0$.

It follows from the first equation of system (1.2) that

$$
\dot{x}(t) \leqslant x(t)\left[\alpha_{1}^{s}-\frac{\alpha_{1}^{i}}{k} x(t)\right] .
$$

Since $\alpha_{1}^{s}>0$, by (i) of Lemma 2.3 and standard comparison theorem, we obtain

$$
\lim _{t \rightarrow+\infty} \sup x(t) \leqslant \frac{k \alpha_{1}^{s}}{\alpha_{1}^{i}} \stackrel{\text { def }}{=} x^{s}>0
$$

Note that $\alpha_{2}^{i} e^{-d_{11} \tau_{1}} \chi^{i}-d_{12}-c_{2}^{s} z_{2}^{s}>0$ implies that $\alpha_{2}^{s} e^{-d_{11} \tau_{1}} x^{s}>\alpha_{2}^{i} e^{-d_{11} \tau_{1}} x^{i}>d_{12}$ holds. Let $\varepsilon>0$ be sufficiently small satisfying $\alpha_{2}^{s} e^{-d_{11} \tau_{1}}\left(\chi^{s}+\varepsilon\right)>d_{12}$. Thus, there exists a $T_{1}>0$ such that

$$
x(t)<x^{s}+\varepsilon, \text { for } t>T_{1} .
$$

We derive from the third equation of system (1.2) that for $t>T_{1}+\tau_{1}$

$$
\dot{y}_{2}(t) \leqslant \alpha_{2}^{s} e^{-d_{11} \tau_{1}}\left(x^{s}+\varepsilon\right) y_{2}\left(t-\tau_{1}\right)-d_{12} y_{2}(t)-\beta_{1}^{i} y_{2}^{2}(t) .
$$

Since $\alpha_{2}^{s} e^{-d_{11} \tau_{1}}\left(\chi^{s}+\varepsilon\right)>d_{12}$, by (i) of Lemma 2.2 and comparison theorem, we acquire that

$$
\lim _{t \rightarrow+\infty} \sup _{2}(t) \leqslant\left[\alpha_{2}^{s} e^{-d_{11} \tau_{1}}\left(x^{s}+\varepsilon\right)-d_{12}\right]\left(\beta_{1}^{i}\right)^{-1} .
$$

Since $\varepsilon>0$ is sufficiently small, we can conclude that

$$
\lim _{t \rightarrow+\infty} \sup _{2}(t) \leqslant\left(\alpha_{2}^{s} e^{-d_{11} \tau_{1}} \chi^{s}-d_{12}\right)\left(\beta_{1}^{i}\right)^{-1} \stackrel{\text { def }}{=} y_{2}^{s}>0
$$

Let $\varepsilon>0$ be sufficiently small satisfying $\alpha_{1}^{\mathfrak{i}}>\mathrm{c}_{1}^{\mathrm{s}}\left(\mathrm{y}_{2}^{\mathrm{s}}+\varepsilon\right)$. Thus, there exists a $\mathrm{T}_{2}>\mathrm{T}_{1}+\tau_{1}$ such that

$$
y_{2}(t)<y_{2}^{s}+\varepsilon, \quad \text { for } t>T_{2} \text {. }
$$

It follows from the first equation of system (1.2) that

$$
\dot{x}(t) \geqslant x(t)\left[\alpha_{1}^{i}-c_{1}^{s}\left(y_{2}^{s}+\varepsilon\right)-\frac{\alpha_{1}^{s}}{k} x(t)\right], \text { for } t>T_{2}+\tau_{1} .
$$

Since $\alpha_{1}^{i}>c_{1}^{s}\left(y_{2}^{s}+\varepsilon\right)$, by (i) of Lemma 2.3 and comparison theorem, we get

$$
\lim _{t \rightarrow+\infty} \inf x(t) \geqslant \frac{k\left[\alpha_{1}^{i}-c_{1}^{s}\left(y_{2}^{s}+\varepsilon\right)\right]}{\alpha_{1}^{s}} .
$$

Since $\varepsilon>0$ is sufficiently small, we obtain that

$$
\lim _{\mathrm{t} \rightarrow+\infty} \inf x(\mathrm{t}) \geqslant k\left(\alpha_{1}^{i}-c_{1}^{\mathrm{s}} y_{2}^{\mathrm{s}}\right)\left(\alpha_{1}^{\mathrm{s}}\right)^{-1} \stackrel{\text { def }}{=} x^{i}>0 .
$$

Substituting (4.3) into the fifth equation of system (1.2) yields

$$
\dot{z}_{2}(t) \leqslant \alpha_{3}^{s} e^{-d_{21} \tau_{2}}\left(y_{2}^{s}+\varepsilon\right) z_{2}\left(t-\tau_{2}\right)-d_{22} z_{2}(t)-\beta_{2}^{i} z_{2}^{2}(t), \quad \text { for } t>T_{2}+\tau_{1} .
$$

Note that $\alpha_{3}^{s} e^{-d_{21} \tau_{2}} y_{2}^{s}>\alpha_{3}^{i} e^{-d_{21} \tau_{2}} y_{2}^{i}>d_{22}$ holds. Let $\varepsilon>0$ be sufficiently small satisfying

$$
\alpha_{3}^{s} e^{-d_{21} \tau_{2}}\left(y_{2}^{s}+\varepsilon\right)>d_{22} .
$$


By (i) of Lemma 2.2 and comparison theorem, it follows that

$$
\lim _{t \rightarrow+\infty} \sup z_{2}(t) \leqslant\left[\alpha_{3}^{s} e^{-d_{21} \tau_{2}}\left(y_{2}^{s}+\varepsilon\right)-d_{22}\right]\left(\beta_{2}^{i}\right)^{-1} .
$$

Since $\varepsilon>0$ is sufficiently small, we acquire that

$$
\lim _{t \rightarrow+\infty} \sup z_{2}(t) \leqslant\left(\alpha_{3}^{s} e^{-d_{21} \tau_{2}} y_{2}^{s}-d_{22}\right)\left(\beta_{2}^{i}\right)^{-1} \stackrel{\text { def }}{=} z_{2}^{s}>0
$$

Let $\varepsilon>0$ be sufficiently small satisfying $\alpha_{2}^{i} e^{-d_{11} \tau_{1}} \chi^{i}>d_{12}+c_{2}^{s}\left(z_{2}^{s}+\varepsilon\right)$. Thus, there exists a $T_{3}>T_{2}+\tau_{1}$ such that $z_{2}(t)<z_{2}^{s}+\varepsilon$ for $t>T_{3}$.

We derive from the third equation of system (1.2) that for $t>T_{3}+\tau_{1}$

$$
\dot{y}_{2}(t) \geqslant \alpha_{2}^{i} e^{-d_{11} \tau_{1}} \chi^{i} y_{2}\left(t-\tau_{1}\right)-\left[d_{12}+c_{2}^{s}\left(z_{2}^{s}+\varepsilon\right)\right] y_{2}(t)-\beta_{1}^{s} y_{2}^{2}(t)
$$

Since $\alpha_{2}^{i} e^{-d_{11} \tau_{1}} \chi^{i}>d_{12}+c_{2}^{s}\left(z_{2}^{s}+\varepsilon\right)$, by (i) of Lemma 2.2 and comparison theorem, we get

$$
\lim _{t \rightarrow+\infty} \inf y_{2}(t) \geqslant\left[\alpha_{2}^{i} e^{-d_{11} \tau_{1}} \chi^{i}-d_{12}-c_{2}^{s}\left(z_{2}^{s}+\varepsilon\right)\right]\left(\beta_{1}^{s}\right)^{-1}, \text { for } t>T_{3}+\tau_{1} .
$$

Since $\varepsilon>0$ is sufficiently small, we can conclude that

$$
\lim _{t \rightarrow+\infty} \inf y_{2}(t) \geqslant\left(\alpha_{2}^{i} e^{-d_{11} \tau_{1}} x^{i}-d_{12}-c_{2}^{s} z_{2}^{s}\right)\left(\beta_{1}^{s}\right)^{-1} \stackrel{\text { def }}{=} y_{2}^{i}>0
$$

Let $\varepsilon>0$ be sufficiently small satisfying $\alpha_{3}^{i} e^{-d_{21} \tau_{2}}\left(y_{2}^{i}-\varepsilon\right)>d_{22}$. Thus, there exists a $T_{4}>T_{3}+\tau_{1}$ such that $y_{2}(t)>y_{2}^{i}-\varepsilon$ for $t>T_{4}$.

We derive from the fifth equation of system (1.2) that for $t>T_{4}+\tau_{2}$

$$
\dot{z}_{2}(\mathrm{t}) \geqslant \alpha_{3}^{i} e^{-d_{21} \tau_{2}}\left(y_{2}^{i}-\varepsilon\right) z_{2}\left(t-\tau_{2}\right)-d_{22} z_{2}(t)-\beta_{2}^{s} z_{2}^{2}(t)
$$

Since $\alpha_{3}^{i} e^{-d_{21} \tau_{2}}\left(y_{2}^{i}-\varepsilon\right)>d_{22}$, by (i) of Lemma 2.2 and comparison theorem, we obtain

$$
\lim _{t \rightarrow+\infty} \inf z_{2}(t) \geqslant\left[\alpha_{3}^{i} e^{-d_{21} \tau_{2}}\left(y_{2}^{i}-\varepsilon\right)-d_{22}\right]\left(\beta_{2}^{s}\right)^{-1} .
$$

Since $\varepsilon>0$ is sufficiently small, we acquire that

$$
\lim _{\mathrm{t} \rightarrow+\infty} \inf z_{2}(\mathrm{t}) \geqslant\left(\alpha_{3}^{i} e^{-\mathrm{d}_{21} \tau_{2}} y_{2}^{i}-\mathrm{d}_{22}\right)\left(\beta_{2}^{s}\right)^{-1} \stackrel{\text { def }}{=} z_{2}^{i}>0 .
$$

For any small positive constant $\varepsilon>0$, it follows from (4.1), (4.2) and (4.4), (4.5), (4.6), (4.7) that there exists a $T_{5}>T_{4}+\tau_{2}$ such that

$$
\begin{aligned}
& x^{i}-\varepsilon<x(t)<x^{s}+\varepsilon, \quad \text { for } t>T_{5}, \\
& y_{2}^{i}-\varepsilon<y_{2}(t)<y_{2}^{s}+\varepsilon, \quad \text { for } t>T_{5}, \\
& z_{2}^{i}-\varepsilon<z_{2}(t)<z_{2}^{s}+\varepsilon, \quad \text { for } t>T_{5} .
\end{aligned}
$$

Note that the second and fourth equations of system (1.2) are equal to the following integral form:

$$
\begin{aligned}
& y_{1}(t)=\int_{t-\tau_{1}}^{t} \alpha_{2}(s) e^{-d_{11}(t-s)} x(s) y_{2}(s) d s \\
& z_{1}(t)=\int_{t-\tau_{2}}^{t} \alpha_{3}(s) e^{-d_{21}(t-s)} y_{2}(s) z_{2}(s) d s
\end{aligned}
$$

Thus, for $t>T_{5}+\tau_{2}$, from (4.8), (4.9) and (4.11), we have

$$
y_{1}(t) \leqslant \int_{t-\tau_{1}}^{t} \alpha_{2}^{s} e^{-d_{11}(t-s)}\left(x^{s}+\varepsilon\right)\left(y_{2}^{s}+\varepsilon\right) d s=\frac{\alpha_{2}^{s}\left(x^{s}+\varepsilon\right)\left(y_{2}^{s}+\varepsilon\right)}{d_{11}}\left(1-e^{-d_{11} \tau_{1}}\right),
$$


and

$$
y_{1}(t) \geqslant \int_{t-\tau_{1}}^{t} \alpha_{2}^{i} e^{-d_{11}(t-s)}\left(x^{i}-\varepsilon\right)\left(y_{2}^{i}-\varepsilon\right) d s=\frac{\alpha_{2}^{i}\left(x^{i}-\varepsilon\right)\left(y_{2}^{i}-\varepsilon\right)}{d_{11}}\left(1-e^{-d_{11} \tau_{1}}\right) .
$$

Setting $\varepsilon \rightarrow 0$ in (4.13) and (4.14) leads to

$$
\begin{aligned}
& \lim _{t \rightarrow+\infty} \sup y_{1}(t) \leqslant \frac{\alpha_{2}^{s} x^{s} y_{2}^{s}}{d_{11}}\left(1-e^{-d_{11} \tau_{1}}\right) \stackrel{\text { def }}{=} y_{1}^{s}, \\
& \lim _{t \rightarrow+\infty} \inf y_{1}(t) \geqslant \frac{\alpha_{2}^{i} x^{i} y_{2}^{i}}{d_{11}}\left(1-e^{-d_{11} \tau_{1}}\right) \stackrel{\text { def }}{=} y_{1}^{i}>0 .
\end{aligned}
$$

By using (4.9), (4.10) and (4.12), similarly to the analysis of (4.13), (4.14), (4.15), (4.16), for $t>T_{5}+\tau_{2}$, we get

$$
\begin{aligned}
& \lim _{t \rightarrow+\infty} \sup z_{1}(t) \leqslant \frac{\alpha_{3}^{s} y_{2}^{s} z_{2}^{s}}{d_{21}}\left(1-e^{-d_{21} \tau_{2}}\right) \stackrel{\text { def }}{=} z_{1}^{s}, \\
& \lim _{t \rightarrow+\infty} \inf z_{1}(t) \geqslant \frac{\alpha_{3}^{i} y_{2}^{i} z_{2}^{i}}{d_{21}}\left(1-e^{-d_{21} \tau_{2}}\right) \stackrel{\text { def }}{=} z_{1}^{i}>0 .
\end{aligned}
$$

By Definition 2.1, we know that the system is permanent. This completes the proof.

Remark 4.2. From Theorem 4.1, we derive bounds for the time delay to ensure that the solutions are permanent. It is natural to ask whether it is possible to obtain tight bounds, i.e., if and only if the time delay is within the range such that the solutions are permanent. However, how to deal with this case is a challenging work, which will be our future study.

Theorem 4.3. Let $\mathrm{n}_{3}=\frac{1}{\mathrm{~d}_{21}} \ln \frac{\alpha_{3}^{s} y_{2}^{s}}{\mathrm{~d}_{22}}$. If $\tau_{1} \in\left(0, \mathrm{n}_{1}\right), \tau_{2} \in\left(\mathrm{n}_{3},+\infty\right)$ and $\alpha_{1}^{\mathrm{i}}-\mathrm{c}_{1}^{\mathrm{s}} \mathrm{y}_{2}^{\mathrm{s}}>0$, then both the prey and predator species are always permanent, and the top predator species will go extinct eventually, that is, the system is partially extinct.

Proof. Let $\left(x(t), y_{1}(t), y_{2}(t), z_{1}(t), z_{2}(t)\right)$ be any positive solution of system (1.2) for $t \geqslant 0$. By $\tau_{2} \in$ $\left(n_{3},+\infty\right)$, one can obtain that $\alpha_{3}^{s} e^{-d_{21} \tau_{2}} y_{2}^{s}-d_{22}<0$ holds. Note that $\alpha_{2}^{i} e^{-d_{11} \tau_{1}} \chi^{i}-d_{12}-c_{2}^{s} z_{2}^{s}>0$, which implies $\alpha_{2}^{i} e^{-d_{11} \tau_{1}} \chi^{i}-d_{12}>0$.

Since $\alpha_{2}^{i} e^{-d_{11} \tau_{1}} \chi^{i}-d_{12}-c_{2}^{s} z_{2}^{s}>0$ and $\alpha_{1}^{i}-c_{1}^{s} y_{2}^{s}>0$, the same arguments as those in the proof of Theorem 4.1 show that (4.1), (4.3) and (4.5) hold, i.e.,

$$
\lim _{t \rightarrow+\infty} \sup x(t) \leqslant x^{s}, \quad \lim _{t \rightarrow+\infty} \inf x(t) \geqslant x^{i}, \quad \lim _{t \rightarrow+\infty} \sup y_{2}(t) \leqslant y_{2}^{s}
$$

Let $\varepsilon>0$ be sufficiently small satisfying $\alpha_{3}^{s} e^{-d_{21} \tau_{2}}\left(y_{2}^{s}+\varepsilon\right)<d_{22}$. Thus, there exists a $T_{6}>0$ such that $y_{2}(t)<y_{2}^{s}+\varepsilon$, for $t>T_{6}$.

It follows from the fifth equation of system (1.2) that

$$
\dot{z}_{2}(t) \leqslant \alpha_{3}^{s} e^{-d_{21} \tau_{2}}\left(y_{2}^{s}+\varepsilon\right) z_{2}\left(t-\tau_{2}\right)-d_{22} z_{2}(t)-\beta_{2}^{i} z_{2}^{2}(t), \quad \text { for } t>T_{6}+\tau_{2} .
$$

Since $\alpha_{3}^{s} e^{-d_{21} \tau_{2}}\left(y_{2}^{s}+\varepsilon\right)<d_{22}$, applying the comparison theorem and the positivity of $z_{2}(t)$ shows that

$$
\lim _{\mathrm{t} \rightarrow+\infty} z_{2}(\mathrm{t})=0 \text {. }
$$

Let $\varepsilon>0$ be sufficiently small satisfying $\alpha_{2}^{i} e^{-d_{11} \tau_{1}} \chi^{i}>d_{12}+\varepsilon$. Thus, there exists a $T_{7}>T_{6}+\tau_{2}$ such that

$$
z_{2}(t)<\frac{\varepsilon}{c_{2}^{s}}, \text { for } \mathrm{t}>\mathrm{T}_{7}
$$

We derive from the third equation of system (1.2) that

$$
\dot{y}_{2}(t) \geqslant \alpha_{2}^{i} e^{-d_{11} \tau_{1}} \chi^{i} y_{2}\left(t-\tau_{1}\right)-\left(d_{12}+\varepsilon\right) y_{2}(t)-\beta_{1}^{s} y_{2}^{2}(t), \quad \text { for } t>T_{7}+\tau_{1} .
$$


Since $\alpha_{2}^{i} e^{-d_{11} \tau_{1}} \chi^{i}>d_{12}+\varepsilon$, by (i) of Lemma 2.2 and comparison theorem, we get

$$
\lim _{t \rightarrow+\infty} \inf y_{2}(t) \geqslant\left[\alpha_{2}^{i} e^{-d_{11} \tau_{1}} \chi^{i}-\left(d_{12}+\varepsilon\right)\right]\left(\beta_{1}^{s}\right)^{-1}
$$

Since $\varepsilon>0$ is sufficiently, we can conclude that

$$
\lim _{t \rightarrow+\infty} \inf y_{2}(t) \geqslant\left(\alpha_{2}^{i} e^{-d_{11} \tau_{1}} \chi^{i}-d_{12}\right)\left(\beta_{1}^{s}\right)^{-1} \stackrel{\text { def }}{=}\left(\hat{y}_{2}\right)^{i}>0 .
$$

For any positive constant $\varepsilon>0$, it follows from (4.17), (4.18), (4.19), (4.20) that there exists a $T_{8}>T_{7}+\tau_{1}$ such that

$$
\begin{gathered}
x^{i}-\varepsilon<x(t)<x^{s}+\varepsilon, \quad \text { for } t>T_{8}, \\
\left(\hat{y_{2}}\right)^{i}-\varepsilon<y_{2}(t)<y_{2}^{s}+\varepsilon, \quad \text { for } t>T_{8}, \\
z_{2}(t)<\varepsilon, \quad \text { for } t>T_{8} .
\end{gathered}
$$

Similarly to the analysis of (4.13), (4.14), (4.15), (4.16), for $t>T_{8}+\tau_{2}$, we derive

$$
\begin{aligned}
& \lim _{t \rightarrow+\infty} \sup y_{1}(t) \leqslant \frac{\alpha_{2}^{s} x^{s} y_{2}^{s}}{d_{11}}\left(1-e^{-d_{11} \tau_{1}}\right), \\
& \lim _{t \rightarrow+\infty} \inf y_{1}(t) \geqslant \frac{\alpha_{2}^{i} x^{i}\left(\hat{y}_{2}\right)^{i}}{d_{11}}\left(1-e^{-d_{11} \tau_{1}}\right) .
\end{aligned}
$$

By using (4.12), (4.21) and (4.22), for $t>T_{8}+\tau_{2}$, we have

$$
z_{1}(t) \leqslant \int_{t-\tau_{2}}^{t} \alpha_{3}^{s} e^{-d_{21}(t-s)}\left(y_{2}^{s}+\varepsilon\right) \varepsilon d s=\frac{\alpha_{3}^{s}\left(y_{2}^{s}+\varepsilon\right) \varepsilon}{d_{21}}\left(1-e^{-d_{21} \tau_{2}}\right)
$$

Setting $\varepsilon \rightarrow 0$ in (4.23) leads to

$$
\lim _{t \rightarrow+\infty} \sup z_{1}(t) \leqslant 0 .
$$

Incorporating into the positivity of $z_{1}(t)$, we get

$$
\lim _{t \rightarrow+\infty} z_{1}(t)=0
$$

This completes the proof.

Remark 4.4. Based on the conditions of Theorem 4.1 and Theorem 4.3 , it is obvious to find that $n_{3}$ is greater than $n_{2}$, thus, one can obtain that longer delay $\tau_{2}$ will lead the top predator species to extinct.

Theorem 4.5. Let $\mathrm{n}_{4}=\frac{1}{\mathrm{~d}_{11}} \ln \frac{\alpha_{2}^{s} x^{\mathrm{s}}}{\mathrm{d}_{12}}$ and $\mathrm{n}_{5}=\frac{1}{\mathrm{~d}_{21}} \ln \frac{\alpha_{3}^{s}}{\mathrm{~d}_{22}}$. If $\tau_{1} \in\left(\mathrm{n}_{4},+\infty\right)$ and $\tau_{2} \in\left(\mathrm{n}_{5},+\infty\right)$, then all predator species will go extinct eventually, and the prey species is always permanent, that is, the system is partially extinct.

Proof. Let $\left(x(t), y_{1}(t), y_{2}(t), z_{1}(t), z_{2}(t)\right)$ be any positive solution of system (1.2) for $t \geqslant 0$. By $\tau_{1} \in\left(n_{4},+\infty\right)$ and $\tau_{2} \in\left(n_{5},+\infty\right)$, it is obvious that $\alpha_{2}^{s} e^{-d_{11} \tau_{1}} \chi^{s}-d_{12}<0$ and $\alpha_{3}^{s} e^{-d_{21} \tau_{2}}-d_{22}<0$ hold.

From the first equation of system (1.2), we conclude that

$$
\dot{x}(t) \leqslant x(t)\left[\alpha_{1}^{s}-\frac{\alpha_{1}^{i}}{k} x(t)\right] .
$$

Since $\alpha_{1}^{\mathrm{s}}>0$, by (i) of Lemma 2.3 and comparison theorem, we obtain that

$$
\lim _{t \rightarrow+\infty} \sup x(t) \leqslant \frac{k \alpha_{1}^{s}}{\alpha_{1}^{i}}=x^{s}
$$

Let $\varepsilon>0$ be sufficiently small satisfying $\alpha_{2}^{s} e^{-d_{11} \tau_{1}}\left(x^{s}+\varepsilon\right)<d_{12}$. Thus, there exists a $T_{9}>0$ such that 
$x(t)<x^{s}+\varepsilon$ for $t>T_{9}$.

We derive from the third equation of system (1.2) that for $t>T_{9}+\tau_{1}$

$$
\dot{y}_{2}(t) \leqslant \alpha_{2}^{s} e^{-d_{11} \tau_{1}}\left(x^{s}+\varepsilon\right) y_{2}\left(t-\tau_{1}\right)-d_{12} y_{2}(t)-\beta_{1}^{i} y_{2}^{2}(t) .
$$

Since $\alpha_{2}^{s} e^{-d_{11} \tau_{1}}\left(x^{s}+\varepsilon\right)<d_{12}$, comparison theorem together with the positivity of $y_{2}(t)$ shows that

$$
\lim _{t \rightarrow+\infty} y_{2}(t)=0 \text {. }
$$

Let $\varepsilon>0$ be sufficiently small satisfying $\alpha_{1}^{i}-\varepsilon>0$. Thus, there exists a $T_{10}>T_{9}+\tau_{1}$ such that

$$
\mathrm{y}_{2}(\mathrm{t})<\frac{\varepsilon}{\mathrm{c}_{1}^{s}}, \text { for } \mathrm{t}>\mathrm{T}_{10} \text {. }
$$

It follows from the first equation of system (1.2) that

$$
\dot{x}(t) \geqslant\left(\alpha_{1}^{i}-\varepsilon\right) x(t)-\frac{\alpha_{1}^{s}}{k} x^{2}(t), \quad \text { for } t>T_{10}+\tau_{1} .
$$

Since $\alpha_{1}^{i}-\varepsilon>0$, by (i) of Lemma 2.3 and comparison theorem, we get

$$
\lim _{t \rightarrow+\infty} \inf x(t) \geqslant k\left(\alpha_{1}^{i}-\varepsilon\right)\left(\alpha_{1}^{s}\right)^{-1} .
$$

Since $\varepsilon>0$ is sufficiently small, we acquire that

$$
\lim _{t \rightarrow+\infty} \inf x(t) \geqslant k \alpha_{1}^{i}\left(\alpha_{1}^{s}\right)^{-1} \stackrel{\text { def }}{=}(\hat{x})^{i}>0 .
$$

Substituting (4.26) into the fifth equation of system (1.2) yields

$$
\dot{z}_{2}(t) \leqslant \frac{\alpha_{3}^{s}}{c_{1}^{s}} e^{-d_{21} \tau_{2}}\left(c_{1}^{s}+\varepsilon\right) z_{2}\left(t-\tau_{2}\right)-d_{22} z_{2}(t)-\beta_{2}^{i} z_{2}^{2}(t), \quad \text { for } t>T_{10}+\tau_{1} .
$$

Let $\varepsilon>0$ be sufficiently small satisfying $\frac{\alpha_{3}^{s}}{c_{1}^{s}} e^{-d_{21} \tau_{2}}\left(c_{1}^{s}+\varepsilon\right)<d_{22}$, comparison theorem together with the positivity of $z_{2}(\mathrm{t})$ shows that

$$
\lim _{\mathrm{t} \rightarrow+\infty} z_{2}(\mathrm{t})=0 \text {. }
$$

For any positive constant $\varepsilon>0$, it follows from (4.24), (4.25), (4.27) and (4.28) that there exists a $\mathrm{T}_{11}>\mathrm{T}_{10}+\tau_{1}$ such that

$$
\begin{gathered}
(\hat{x})^{i}-\varepsilon<x(t)<x^{s}+\varepsilon, \quad \text { for } t>T_{11}, \\
y_{2}(t)<\varepsilon, \quad \text { for } t>T_{11}, \\
z_{2}(t)<\varepsilon, \quad \text { for } t>T_{11} .
\end{gathered}
$$

Thus, for $t>T_{11}+\tau_{1}$, from (4.11), (4.29) and (4.30), we have

$$
\mathrm{y}_{1}(\mathrm{t}) \leqslant \frac{\alpha_{2}^{\mathrm{s}}\left(\mathrm{x}^{\mathrm{s}}+\varepsilon\right) \varepsilon}{\mathrm{d}_{11}}\left(1-e^{-\mathrm{d}_{11} \tau_{1}}\right) .
$$

Setting $\varepsilon \rightarrow 0$ in (4.32) leads to

$$
\lim _{t \rightarrow+\infty} \sup y_{1}(t) \leqslant 0 .
$$

Incorporating into the positivity of $y_{1}(t)$, we obtain that

$$
\lim _{t \rightarrow+\infty} y_{1}(t)=0 \text {. }
$$

By using (4.12), (4.30) and (4.31), similarly to the analysis of (4.32), (4.33), (4.34), we get

$$
\lim _{\mathrm{t} \rightarrow+\infty} z_{1}(\mathrm{t})=0 \text {. }
$$

This completes the proof. 
Remark 4.6. Let $n=\max \left\{n_{3}, n_{5}\right\}$, if $\tau_{1} \in\left(0, n_{1}\right), \tau_{2} \in(n,+\infty)$ and $\alpha_{1}^{i}-c_{1}^{s} y_{2}^{s}>0$, then it is easy to obtain that the conditions of Theorem 4.3 hold. According to Theorem 4.3, both the prey and predator species are permanent, and the top predator species will be driven to extinction. On the other hand, if $\tau_{1} \in\left(n_{4},+\infty\right)$ and $\tau_{2} \in(n,+\infty)$, then the conditions of Theorem 4.5 hold. Therefore, all predator species will go to extinction, and the prey species is permanent.

Remark 4.7. From Theorem 4.3 and Theorem 4.5 , it is to see that $n_{4}$ is greater than $n_{1}$. By Remark 4.6 , one can obtain that longer delay $\tau_{1}$ will lead the predator species to extinct.

\section{An example and numerical simulations}

In this section, one example is presented to demonstrate the correctness and effectiveness of the obtained results.

Example 5.1. Consider the following system with two different time delays

$$
\left\{\begin{array}{l}
\dot{x}(t)=x(t)\left[20\left(1-\frac{x(t)}{2}\right)-2 y_{2}(t)\right] \\
\dot{y}_{1}(t)=6 x(t) y_{2}(t)-\ln 2 y_{1}(t)-6 e^{-\ln 2 \tau_{1}} x\left(t-\tau_{1}\right) y_{2}\left(t-\tau_{1}\right) \\
\dot{y}_{2}(t)=6 e^{-\ln 2 \tau_{1}} x\left(t-\tau_{1}\right) y_{2}\left(t-\tau_{1}\right)-y_{2}(t)-\left(\frac{3}{2}+\frac{1}{2} \cos t\right) y_{2}^{2}(t)-y_{2}(t) z_{2}(t) \\
\dot{z}_{1}(t)=16 y_{2}(t) z_{2}(t)-\ln 2 z_{1}(t)-16 e^{-\ln 2 \tau_{2}} y_{2}\left(t-\tau_{2}\right) z_{2}\left(t-\tau_{2}\right) \\
\dot{z}_{2}(t)=16 e^{-\ln 2 \tau_{2}} y_{2}\left(t-\tau_{2}\right) z_{2}\left(t-\tau_{2}\right)-z_{2}(t)-(15+\cos t) z_{2}^{2}(t)
\end{array}\right.
$$

where $\tau_{1}>0$ and $\tau_{2}>0$ are constant time delay.

Case I. Let $\tau_{1}=1$ and $\tau_{2}=2$.

By some simple calculations, we obtain $n_{1} \approx 1.348, n_{2} \approx 2.363$ and $\alpha_{1}^{i}-c_{1}^{s} y_{2}^{s}=10>0$. It is easy to find that $\tau_{1} \in\left(0, n_{1}\right), \tau_{2} \in\left(0, n_{2}\right)$. Thus, the conditions of Theorem 4.1 hold and system (5.1) is permanent. The numerical simulation is shown in Figure 1.

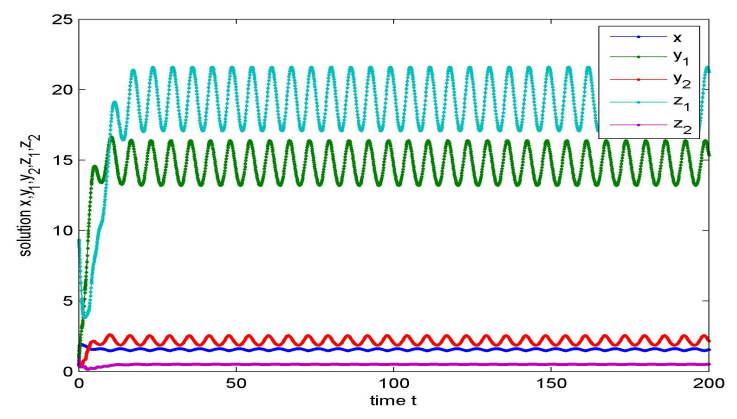

Figure 1: The temporal solution found by numerical integration of system (5.1) with $\tau_{1}=1, \tau_{2}=2$ and

$$
\left(\phi(\theta), \varphi_{1}(\theta), \varphi_{2}(\theta), \psi_{1}(\theta), \psi_{2}(\theta)\right)=\left(0.4, \frac{18}{25 \ln 2}, 0.6, \frac{162}{25 \ln 2}, 0.9\right) .
$$

Case II. Let $\tau_{1}=1$ and $\tau_{2}=9$.

By some simple calculations, we know $n_{1} \approx 2.585, n_{3} \approx 3.689$ and $\alpha_{1}^{i}-c_{1}^{s} y_{2}^{s}=10>0$. It is obvious to see $\tau_{1} \in\left(0, n_{1}\right)$ and $\tau_{2} \in\left(n_{3},+\infty\right)$. According to Theorem 4.3, we can show that the prey and the predator species will be permanent, and the top predator species will go to extinction. The numerical simulation illustrates our result (see Figure 2). 


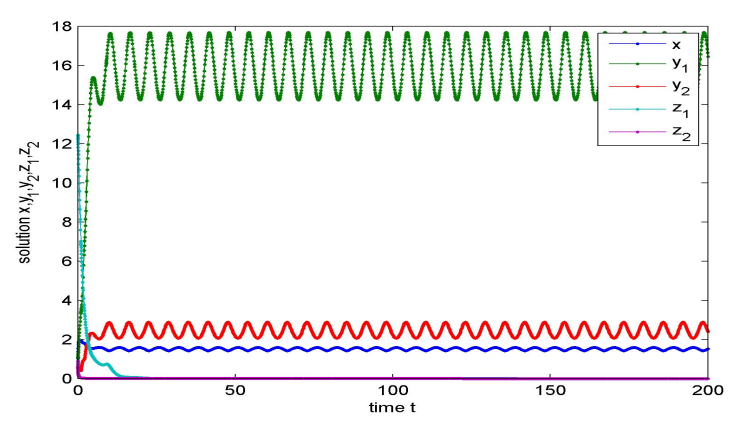

Figure 2: The temporal solution found by numerical integration of system (5.1) with $\tau_{1}=1, \tau_{2}=9$ and $\left(\phi(\theta), \varphi_{1}(\theta), \varphi_{2}(\theta), \psi_{1}(\theta), \psi_{2}(\theta)\right)=\left(0.4, \frac{18}{25 \ln 2}, 0.6, \frac{208}{25 \ln 2} \times\left(1-\frac{1}{2^{9}}\right), 0.9\right)$.

Case III. Let $\tau_{1}=16$ and $\tau_{2}=9$.

By the simple calculation, we obtain $n_{4} \approx 3.585$ and $n_{5}=4$. Obviously, $\tau_{1}$ and $\tau_{2}$ satisfy assumptions $\tau_{1} \in\left(n_{4},+\infty\right)$ and $\tau_{2} \in\left(n_{5},+\infty\right)$. It follows from Theorem 4.5 , the prey species will be permanent, and the predator and the top predator species will go to extinction. The numerical simulations also confirm this phenomenon (see Figure 3).

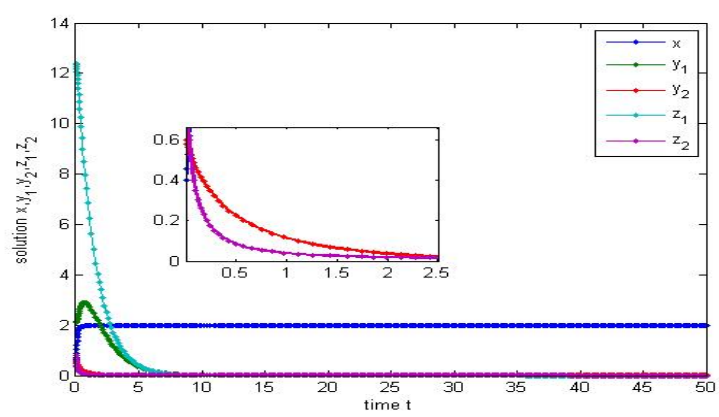

Figure 3: The temporal solution found by numerical integration of system (5.1) with $\tau_{1}=16, \tau_{2}=9$ and $\left(\phi(\theta), \varphi_{1}(\theta), \varphi_{2}(\theta), \psi_{1}(\theta), \psi_{2}(\theta)\right)=\left(0.4, \frac{36}{25 \ln 2} \times\left(1-\frac{1}{2^{16}}\right), 0.6, \frac{208}{25 \ln 2} \times\left(1-\frac{1}{2^{9}}\right), 0.9\right)$.

\section{Conclusion}

Incorporating two delays, a new delayed three-species food chain model with stage-structure and time-varying coefficients is established in this paper. The positivity and boundedness of solutions of the model have been proved. Some sufficient conditions on the permanence and partial extinction of system are derived by the comparison theorem. From Theorem 4.1, we get the sufficient conditions: $\tau_{1} \in\left(0, n_{1}\right)$, $\tau_{2} \in\left(0, n_{2}\right)$ and $\alpha_{1}^{i}-c_{1}^{s} y_{2}^{s}>0$ for the permanence of system (1.2). According to Remark 4.6, we can obtain the following conclusions for partial extinction of system (1.2): the prey and predator species will coexist and the top predator species will go extinct eventually if $\tau_{1} \in\left(0, n_{1}\right), \tau_{2} \in(n,+\infty)$ and $\alpha_{1}^{i}-c_{1}^{s} y_{2}^{s}>0$; all the predators will go extinct and the prey species persists if $\tau_{1} \in\left(n_{4},+\infty\right)$ and $\tau_{2} \in(n,+\infty)$. Our study demonstrates that the delays have great impacts on the permanence for the system. More precisely, according to Remarks 4.4 and 4.7, longer delay $\tau_{2}$ will lead the top predator species to extinct; longer delay $\tau_{1}$ will lead the all predators to extinct.

The obtained results in this paper may provide some new insights for predicting the dynamical behaviors of the food chain system and protecting the ecological balance in a real ecosystem. In addition, we would like to point out that it is more detailed to analysis the existence and stability of periodic solutions of system (1.2) if the coefficients in system (1.2) are $\omega$-periodic in $t$. We leave them for our future work. 


\section{Acknowledgment}

We are extremely grateful to one anonymous reviewer and the editor for their valuable comments and suggestions which have contributed considerably to the improved presentation of this paper.

This work is supported partially by the National Natural Science Foundation of China (Nos. 71471020, 11771059), Hunan Provincial Natural Science Foundation (No. 2016JJ1001), Scientific Research Fund of Hunan Provincial Education Department (No. 15A003), and Hunan Provincial and CSUST Innovation Foundation for Postgraduate (Nos. CX2017B490, CX2017SS23).

\section{References}

[1] W. G. Aiello, H. I. Freedman, A time delay model of single-species growth with stage structure, Math. Biosci., 101 (1990), 139-153. 1

[2] H. Baek, H. H. Lee, Permanence of a three-species food chain system with impulsive perturbations, Kyungpook Math. J., 48 (2008), 503-514. 1

[3] A. A. Berryman, The Orgins and Evolution of Predator-Prey Theory, Ecology, 73 (1992), 1530-1535. 1

[4] H. Boudjellaba, T. Sari, Stability loss delay in harvesting competing populations, J. Differential Equations, 152 (1999), 394-408. 1

[5] K. Charles Kendeigh, Animal Ecology, Prentice-Hall, New York, (1961). 1

[6] F. Chen, H. Wang, Y. Lin, W. Chen, Global stability of a stage-structured predator-prey system, Appl. Math. Comput., 223 (2013), 45-53. 1

[7] J. Cui, L. Chen, W. Wang, The effect of dispersal on population growth with stage-structure, Comput. Math. Appl., 39 (2000), 91-102. 1

[8] J. M. Cushing, Periodic time-dependent predator-prey system, SIAM J. Appl. Math., 32 (1977), 82-95. 1

[9] K. Das, K. Reddy, M. Srinivas, N. Gazi, Chaotic dynamics of a three species prey-predator competition model with noise in ecology, Appl. Math. Comput., 231 (2014), 117-133. 1

[10] L. Deng, X. Wang, M. Peng, Hopf bifurcation analysis for a ratio-dependent predator-prey system with two delays and stage structure for the predator, Appl. Math. Comput., 231 (2014), 214-230. 1

[11] T. Faria, Stability and bifurcation for a delayed predator-prey model and the effect of diffusion, J. Math. Anal. Appl., 254 (2001), 433-463. 1

[12] W. Fengying, W. Ke, Permanence of variable coefficients predator-prey system with stage structure, Appl. Math. Comput., 180 (2006), 594-598. 1

[13] X. He, Stability and delays in a predator-prey system, J. Math. Anal. Appl., 198 (1996), 355-370. 1

[14] H. Hu, L. Huang, Stability and Hopf bifurcation in a delayed predator-prey system with stage structure for prey, Nonlinear Anal. Real World Appl., 11 (2010), 2757-2769. 1

[15] G. Hu, X. Li, Stability and Hopf bifurcation for a delayed predator-prey model with disease in the prey, Chaos Solitons Fractals, 45 (2012), 229-237. 1

[16] C. Huang, J. Cao, Convergence dynamics of stochastic Cohen-Grossberg neural networks with unbounded distributed delays, IEEE Trans. Neural Netw., 22 (2011), 561-572. 1

[17] C. Huang, J. Cao, J. Cao, Stability analysis of switched cellular neural networks: A mode-dependent average dwell time approach, Neural Netw., 82 (2016), 84-99.

[18] C. Huang, C. Peng, X. Chen, F. Wen, Dynamics analysis of a class of delayed economic model, Abstr. Appl. Anal., 2013 (2013), 12 pages.

[19] C. Huang, Z. Yang, T. Yi, X. Zou, On the basins of attraction for a class of delay differential equations with non-monotone bistable nonlinearities, J. Differential Equations, 256 (2014), 2101-2114. 1

[20] T. K. Kar, S. Jana, Stability and bifurcation analysis of a stage structured predator-prey model with time delay, Appl. Math. Comput., 219 (2012), 3779-3792. 1

[21] Y. Kuang, J. W.-H. So, Analysis of a delayed two-stage population with space-limited recruitment, SIAM J. Appl. Math., 55 (1995), 1675-1696. 1

[22] S. Liu, B. Kouche, N.-E. Tatar, Permanence extinction and global asymptotic stability in a stage structured system with distributed delays, J. Math. Anal. Appl., 301 (2005), 187-207. 1

[23] Z.-H. Ma, Z.-Z. Li, S.-F. Wang, T. Li, F.-P. Zhang, Permanence of a predator-prey system with stage structure and time delay, Appl. Math. Comput., 201 (2008), 65-71. 2.3

[24] W. Mbava, J. Y. T. Mugisha, J. W. Gonsalves, Prey, predator and super-predator model with disease in the super-predator, Appl. Math. Comput., 297 (2017), 92-114. 1

[25] B. Patra, A. Maiti, G. Samanta, Effect of time-delay on a ratio-dependent food chain model, Nonlinear Anal. Model. Control, 14 (2009), 199-216. 1

[26] S. Ruan, Y. Tang, W. Zhang, Versal unfoldings of predator-prey systems with ratio-dependent functional response, J. Differential Equations, 249 (2010), 1410-1435. 1 
[27] C. Shen, M. You, Permanence and extinction of a three-species ratio-dependent food chain model with delay and prey diffusion, Appl. Math. Comput., 217 (2010), 1825-1830. 1

[28] Z. Teng, Y. Yu, The extinction in nonautonomous prey-predator Lotka-Volterra systems, Acta Math. Appl. Sinica, 15 (1999), 401-408. 1

[29] B. Tian, S. Zhong, Z. Liu, Extinction and persistence of a nonautonomous stochastic food-chain system with impulsive perturbations, Int. J. Biomath., 2016 (2016), 26 pages. 1

[30] K. Wang, Permanence and global asymptotical stability of a predator-prey model with mutual interference, Nonlinear Anal. Real World Appl., 12 (2011), 1062-1071. 1

[31] W. Wang, G. Mulone, F. Salemi, V. Salone, Permanence and stability of a stage-structured predator-prey model, J. Math. Anal. Appl., 262 (2001), 499-528. 1

[32] R. Xu, M. A. J. Chaplain, F. A. Davidson, Global stability of Lotka-Volterra type predator-prey model with stage structure and time delay, Appl. Math. Comput., 159 (2004), 863-880. 1, 2.2

[33] C. Xu, S. Yuan, T. Zhang, Global dynamics of a predator-prey model with defense mechanism for prey, Appl. Math. Lett., 62 (2016), 42-48. 1

[34] P. Yongzhen, M. Guo, C. Li, A delay digestion process with application in a three-species ecosystem, Commun. Nonlinear Sci. Numer. Simul., 16 (2011), 4365-4378. 1

[35] H. Zhang, L. Chen, R. Zhu, Permanence and extinction of a periodic predator-prey delay system with functional response and stage structure for prey, Appl. Math. Comput., 184 (2007), 931-944. 1 